\title{
Les technologies numériques dans les pays en développement. Quel paradigme?
}

Digital technologies in developing countries. What paradigm?

Tecnologías digitales en países en desarrollo. Qué ¿paradigma?

\section{Ahmed Dahmani}

\section{CpenEdition}

\section{Journals}

Édition électronique

URL : http://journals.openedition.org/ctd/437

DOI : $10.4000 /$ ctd. 437

ISSN : 2491-1437

Éditeur

Chaire Unesco Pratiques émergentes en technologies et communication pour le développement

Référence électronique

Ahmed Dahmani, « Les technologies numériques dans les pays en développement. Quel paradigme? », Communication, technologies et développement [En ligne], 6 | 2018, mis en ligne le 18 décembre 2018, consulté le 10 décembre 2020. URL : http://journals.openedition.org/ctd/437 ; DOI : https://doi.org/10.4000/ctd.437

Ce document a été généré automatiquement le 10 décembre 2020.

Communication, technologies et développement 


\title{
Les technologies numériques dans les pays en développement. Quel paradigme?
}

\author{
Digital technologies in developing countries. What paradigm? \\ Tecnologías digitales en países en desarrollo. Qué ¿paradigma?
}

\section{Ahmed Dahmani}

1 Les pays en développement (PED), notamment en Afrique, connaissent une croissance considérable des technologies numériques $(\mathrm{TN})^{1}$ lors de la dernière décennie. Balbutiante et hésitante à ses débuts, mais toujours inégale, la progression de leurs utilisateurs dépasse toutes les prévisions et suscite tantôt incrédulité tantôt espoir de développement. Il est vrai que l'engouement des sociétés africaines pour ces technologies est réel et leurs capacités d'appropriation pas moins. Elles s'accrochent, certes avec beaucoup d'obstacles et de handicaps, au mouvement planétaire de la troisième révolution industrielle ${ }^{2}$, la révolution numérique. Portée par cette innovation capitale de ces dernières décennies, les $\mathrm{TN}$, cette révolution est fondée sur la maitrise de l'information, l'accès au savoir et la capacité de chacun à interagir avec le reste du monde (Rifkin, 2011). Les TN se sont progressivement imposées au point qu'il n'est plus possible d'envisager des formes de travail, d'habitat, de loisirs, etc., qui puissent en faire l'économie. Elles ont, en effet, radicalement transformé les modes et le sens même des vies des individus à travers la planète.

2 La révolution numérique qui n'épargne pas les PED et encore moins l'Afrique a réactivé le mythe du millénarisme technologique banalisant de fait cette technologie (1re partie). Pourtant, les TN se distinguent bien des technologies traditionnelles et notamment de la chaîne des médias classiques (presse, radio, TV). Elles nous incitent à la nécessaire rupture épistémologique, à l'élaboration d'un nouveau paradigme qui intègre les effets socioéconomiques et politiques singuliers que ces technologies provoquent dans les PED (2e partie). 


\section{La révolution numérique irradie aussi l'Afrique}

3 Le rythme et l'ampleur des innovations dans le monde des TN sont inédits dans l'histoire humaine. Ils ont affecté les modèles économiques, les modes de consommation et les différentes formes d'échange. En moins de vingt ans - le premier ordinateur personnel date du milieu des années 80 - elles ont radicalement transformé les modes de vie, les façons de produire, les rapports au temps et à l'espace, l'environnement culturel des pays développés (PD). Ils participent et accélèrent le processus de globalisation irradiant ainsi de leurs effets tous les espaces et sociétés de la planète comme le montre l'exemple africain.

\section{L'Afrique a sauté une génération technique}

Les indicateurs concernant l'utilisation des TN sont impressionnants. En 2015, 43,4 \% de la population mondiale utilise Internet et sur ces quelque 3,2 milliards d'individus, nombre qui a presque doublé en cinq ans, près de 2 milliards se connectent en mouvement, grâce aux smartphones et aux tablettes. À l'horizon 2020 (programme connect 2020) ${ }^{3}, 56 \%$ de l'humanité sera alors en prise directe avec l'Internet, via des terminaux intelligents, fixes ou mobiles, eux- mêmes en connexion avec plus de 50 milliards d'objets communiquant avec les humains ou entre eux.

5 La mondialisation des TN qu'ils contribuent à accélérer et à redessiner en permanence est aujourd'hui un fait établi. L'adhésion quasi planétaire à ces technologies, la massification de leurs usages, l'investissement "par des populations de plus en plus hétérogènes géographiquement, socialement et culturellement » (Cardon, 2010) sont singuliers à l'échelle de l'histoire humaine. Les TN présentent en fait cette caractéristique qu'aucune technologie n'a pu transcender complètement, celui d'atténuer, en se jouant des frontières, des distinctions traditionnelles.

6 La première date de la fin du 18e siècle et se fonde sur les progrès en mécanique et en chimie. La seconde apparait vers la fin du 19e siècle avec la maitrise de l'énergie et l'invention du moteur à explosion. Les deux vont provoquer des séries de transformations socioéconomiques et politiques qui vont profondément bouleverser le monde occidental.

7 Ce qui conforte ce constat largement partagé aujourd'hui: «le numérique dévore le monde » (Andreessen, 2011). Avec une offre d'information amplifiée et une multiplication des opportunités pour y accéder, les nouveaux outils numériques participent, souvent de manière décisive, aux nouvelles façons d'appréhender et de penser notre monde. Ainsi, «avec l'informatique et les réseaux, ce ne sont pas les supports qui changent, c'est beaucoup plus fondamentalement l'ontogenèse de la pensée, sa manière de se produire, de se disséminer et en somme, d'être (Mathias, 2011).»

Les pays en développement (PED) ne sont donc pas en reste. Les pays africains, notamment, connaissent un dynamisme singulier dans l'adoption de ces TN toutes ces dernières années. Selon l'indice de développement des TN (IDI) ${ }^{4}$ établi par l'UIT, l'Afrique a une valeur moyenne de 2,64 points, soit à peine plus de la moitié de la moyenne mondiale $(5,11)$ et le premier pays du continent, Maurice, avec un IDI de 5,88 est classé à la 72ème place sur 176 en 2017. Les dernières places du classement regroupent la quasimajorité du continent. Cependant, en dépit de la double fracture digitale et cognitive qui 
la caractérise depuis l'avènement des TN dans les années $1990^{5}$, l'Afrique connaît une croissance impressionnante de ces technologies par comparaison avec les pays développés ${ }^{6}$. Cependant, en dépit de la double fracture digitale et cognitive qui la caractérise depuis l'avènement des TN dans les années 1990, l'Afrique connaît une croissance impressionnante de ces technologies - que doit tempérer l'effet rattrapage par comparaison avec les pays développés où certains segments des TN sont en voie de stabilisation, voire même de stagnation. Certaines firmes multinationales du secteur espèrent bien faire de l'Afrique le nouvel espace de croissance de leurs activités. Il est vrai que si dans ces derniers certains segments des TN se situent dans une phase de stabilisation, voire de stagnation, l'Afrique constitue un véritable réservoir de croissance pour les firmes du secteur (Chéneau Loquay, 2010) doit tempérer l'effet rattrapage. Il est indéniable aussi que cette évolution est renforcée par l'engouement sans cesse renouvelé des Africains, y compris ruraux, pour ces technologies. L'évolution des connexions à Internet est remarquable. Entre 2005 et 2015, le nombre de ménages accédant à Internet a été multiplié par plus de six, alors que le total monde l'a été par plus de deux. Plus intéressant encore, si la part de l'Afrique dans le monde demeure particulièrement faible, le pourcentage d'individus connectés en Afrique $(20,7 \%)$ est supérieur au pourcentage de ménages connectés $(10,7)$, tandis que l'on observe plutôt l'inverse pour les pays développés (77,6 \% d'individus contre 82,1 \% de ménages).

Mais, c'est la téléphonie mobile qui a connu une adhésion populaire particulièrement impressionnante. En 2015, l'Afrique compte près de 700 millions d'utilisateurs pour une population d'environ 1,2 milliard. Et avec un taux moyen de progression de $7 \%$, ce chiffre dépassera le milliard en 2021. Entre 2005 et 2015, le nombre des utilisateurs a été multiplié par près de cinq alors que le total monde a été multiplié par moins de trois. La part de l'Afrique dans le monde est passée de 3,9\% en 2005 à 17,4\% en 2015 pour le mobile haut débit (78 \% pour l'Europe et les USA). Dans une grande partie de ce continent, la plupart des utilisateurs du cellulaire n'ont jamais disposé d'un téléphone fixe. Ils sont passés de rien à la communication tous azimuts. C'est ainsi que les réseaux mobiles se sont imposés comme des alternatives au fixe dans des régions qui étaient jusque-là sans connexion. Le continent saute ainsi une génération technique, celle du filaire, en avance sur les continents développés où cette tendance demeure incertaine. Entre 2005 et 2015, la télédensité du fixe stagne à 1,5 alors que celle du mobile est passée de 12,4 à 63 . De nombreux facteurs expliquent cette progression et cet engouement : facilité d'utilisation, mobilité et flexibilité dans le déploiement, qualité et diversité des services, etc. Au plan financier, le système de prépaiement ${ }^{7}$, le transfert de crédit, la mise à disposition gratuite ou peu chère de la carte SIM se révèlent bien adaptés au budget limité des ménages. Pourtant, les tarifs sont parmi les plus chers pratiqués dans le monde ${ }^{8}$ permettant ainsi aux opérateurs d'engranger de substantiels bénéfices.

\section{Sortir du déterminisme technologique}

10 Le développement par la technologie est un thème récurrent dans les discours développementistes. Il a été largement partagé entre la fin de la Seconde Guerre mondiale et celle des années 1970. De nombreux projets de développement prenaient appui sur la croyance dans un progrès technique qui permettrait aux PED d'accélérer leur mouvement vers le développement, voire d'en court-circuiter des étapes?. 
11 Cet optimisme technologique qu'exprime le «leapfrogging» ou comment bruler les étapes du développement, n'est contrarié ici que par la fracture numérique qu'il faut combler au prix d'une mobilisation internationale et, surtout, d'une libéralisation du marché des télécommunications. Dès les années 90 les conférences, les programmes et les rapports sur le thème TIC et développement se multiplient sous l'égide des grands forums et organisations internationaux (ONU, Banque mondiale, G8, Davos, USAid, Union européenne, UNESCO, UIT, etc.). Ainsi, le PNUD manifeste le même enthousiasme dans son rapport sur le développement humain de 1999 (PNUD, 1999). Il y souligne la nécessité de mobiliser les capitaux pour que la société de l'information soit équitablement partagée, allant jusqu'à suggérer l'idée d'instaurer une « taxe sur les bits », à l'image de la taxe « Tobin » sur les transactions financières, qui reviendrait à prélever un montant très modeste calculé sur le volume des données transmises via Internet.

12 À l'appui de cette assertion, la mise en avant des nombreuses expériences montrant comment certaines opportunités économiques ont pu se développer grâce notamment à la réduction des coûts de transaction et la baisse des coûts des services de télécommunications. Les exemples mis en avant son nombreux : cybercafés, boutiques de téléservices, entreprises de maintenance ou de production d'applications et contenus adaptés aux besoins locaux, délocalisations de services comme les centres d'appels, le secrétariat, l'édition, les saisies de données, les services bancaires et d'assurances, les services informatiques, etc.

La généralisation de ces expériences serait la nouvelle voie miracle pour le développement des pays du sud ${ }^{10}$. Ignorant le fait que ces pays se caractérisent par l'absence ou l'inefficience de capacités technologiques qui les empêchent de participer directement ou indirectement à leur développement. De même que la rapidité du rythme des innovations technologiques rend encore plus improbable une quelconque participation des pays d'Afrique à «l'économie de la connaissance» tant la fracture cognitive demeure considérable ${ }^{11}$.

Pour autant, les TN, du fait même de leur nature, de leur vitesse de propagation et de leur accès relativement facilité nous invitent à renouveler nos outils conceptuels notamment en matière de développement.

\section{Pour un nouveau paradigme des TN dans les PED}

15 La consommation du segment mobile des TN est croissante en Afrique même si le taux de pauvreté est parmi les plus élevés au monde. Elle se distingue de celle des autres services par le fait qu'elle ne constitue pas un acte individuel, isolé, mais collectif, en réseau. Consommer des services mobiles est une mise en relation avec les autres, voire avec le monde quand on a l'opportunité de posséder un smartphone et une connexion Internet. Et à ce titre, le cellulaire permet à de plus en plus d'individus de communiquer, de s'informer, voire de se former. Les exemples en Afrique foisonnent à ce sujet démontrant ainsi ce souhait voire cette quête de l'individu vers plus d'autonomie et de liberté dans un monde de plus en plus connecté. 


\section{Singularités africaines ?}

16 L'intérêt porté par les Africains pour les TN épouse les mêmes contours que ceux observés dans les pays développés. Toutes les études indiquent que l'âge est un facteur clé de l'usage de ces technologies, les jeunes ayant tendance à les adopter plus facilement. Et à ce titre, l'Afrique présente des potentialités de développement des TN supérieures aux autres régions du monde. Le continent sera le plus jeune au monde à partir de 2050 et le deuxième le plus peuplé. Le pourcentage de la population des moins de quinze ans représente près de $40 \%$ du total, soit environ le double des pays développés. Les opérateurs locaux comme étrangers ont déjà anticipé l'afflux de nouveaux utilisateurs se procurant le budget nécessaire pour acquérir un mobile et un crédit d'appel même si les prix, dans la plupart des pays africains, demeurent parmi les plus élevés au monde. La libéralisation, avec un bilan contrasté, a bien impulsé une dynamique de concurrence qui a fait baisser les prix de $25 \%$ entre 2008 et 2010 , contre 18 et $15 \%$ respectivement dans la région Asie-Pacifique et les États arabes, d'environ $11 \%$ dans la région Amérique et de $15 \%$ en Europe. Mais ils demeurent encore trop élevés. En 2013, selon la Banque mondiale

${ }^{12}$, le prix des services du téléphone mobile classiques dans le pays le plus cher (situé sur le continent africain) représentait cinquante fois le prix des mêmes services dans le pays le meilleur marché parmi les PD. Pour le haut débit, les tarifs varient d'un à cent. Ces tarifs grèvent ainsi lourdement les revenus de la majorité des ménages africains. Selon l'UIT,

« en dépit de la baisse des tarifs de la large bande mobile dans les pays les moins avancés (PMA) [en majorité africains, AD], les prix en pourcentage de revenu/ habitant sont en moyenne deux fois plus élevés dans ces pays que la moyenne de tous les PED et vingt fois plus élevés que dans les PD $»^{13}$.

17 L'engouement persistant pour le mobile interpelle la théorie classique du consommateur. Nous sommes loin de ce " consommateur rationnel souverain » qui cherche à maximiser son utilité en tenant compte des contraintes de revenu et de prix. Dans le cas qui nous occupe, ces contraintes ne semblent nullement dissuader l'utilisateur africain dans sa volonté d'accès à ces technologies et à leurs applications même si les besoins de base ne sont pas satisfaits. Le même rapport de la Banque mondiale signale que dans les PED, les ménages qui possèdent un mobile sont plus nombreux que ceux qui ont accès à l'électricité ou à de l'eau salubre. De même que l'« effet Veblen » qui met en avant la logique de la consommation ostentatoire ou l'effet d'imitation et de distinction n'épuise pas plus le sujet. Le mobile fait partie aujourd'hui du quotidien des individus et des sociétés ${ }^{14}$. Il ne peut être réduit à un objet d'apparat ou de distinction ${ }^{15}$. Il répond à des besoins et à des habitudes de consommation différents de ceux des PD poussant les opérateurs à ajuster leur offre. Et face à la défectuosité des réseaux, certains usagers peuvent posséder deux téléphones voire plus ou alors un appareil avec une double carte SIM afin d'être connecté sur le réseau qui fonctionne ou alors pour profiter d'une tarification plus avantageuse d'un opérateur. Il constitue une source régulière d'innovation sociale permettant aux individus de gagner en liberté en leur offrant « des capacités d'invention collective, donc de désobéir, de créer, de relier, de trouver des solutions efficaces (Kiyindou, 2015) » et d'élaborer des dispositifs de contre-pouvoir.

Le développement impressionnant du cellulaire ${ }^{16}$, de plus en plus convergent avec l'Internet, semble donc marquer une tournure singulière, en ce sens que son arrivée dans les années 2000 a été un accélérateur considérable de l'appropriation des TN en Afrique, 
similaire à ce que fut l'irruption des ordinateurs personnels dans les pays développés. Il s'accompagne d'une multiplicité de services, de manières d'utilisation, de détournement de prescriptions imposées par les opérateurs ou les fabricants et qui est le propre d'une innovation comme " résultat d'une action des utilisateurs ... sur une échelle plus large » (Mezouaghi, 2007: 62), une innovation par l'usage (Cardon, op. cité). Comme si l'utilisateur africain agissait comme un «producteur » de nouveaux produits, mais aussi de manières d'utiliser les outils auxquels il accède et dont doivent tenir compte les fabricants à l'avenir ${ }^{17}$. Le mobile, qui n'est plus réservé aux seuls détenteurs de moyens financiers et culturels pour son utilisation, a profondément pénétré les sociétés dans ses différentes strates sociales, sa diversité spatiale et même dans des zones rurales souséquipées ou dépourvues des infrastructures de base (télécommunications et électricité). Il est devenu un outil domestique courant, voire indispensable, dans la vie et la culture quotidiennes s'inscrivant dans la vie sociale des individus et des groupes qui peuvent ainsi les utiliser pour une redéfinition des enjeux socioculturels et politiques.

\section{Promotion de l'individu et quête d'ouverture}

Grâce aux TN, qui favorisent les relations horizontales, certaines hiérarchies et relations de pouvoir sont de plus en plus chahutées. Les plus jeunes, profitant de leur maitrise des diverses fonctionnalités des outils, osent s'exprimer avec plus de liberté, et sans contester frontalement le pouvoir des aînés, réduisent leur traditionnel contrôle ${ }^{18}$. En milieu urbain et encore plus en milieu rural, les nouveaux rôles que les TN permettent de jouer contribuent à redéfinir la place des individus au sein de leurs communautés d'origine. De même que dans des sociétés qui se caractérisent par la prégnance des structures communautaires, notamment la famille, les TN permettent l'affirmation de l'individu en dehors de ces communautés, mais sans nécessairement s'y opposer. Les femmes, jeunes et moins jeunes, ont désormais la possibilité de communiquer hors des contraintes sociales. Leurs paroles sont de moins en moins retenues et elles peuvent mener deux vies parallèles, disjointes : l'une dans la tradition familiale, l'autre dans l'ouverture, voire dans la modernité qui peut aboutir à des liens réels pouvant aller jusqu'au mariage sans que les familles se soient concertées ou mêlées en amont.

Par ailleurs, la technologie mobile tend à redéfinir les distinctions et les découpages traditionnels : interne/externe, local/global, urbain/rural, espace public/espace privé. Comme jamais auparavant, elle facilite la circulation de l'information dans les villages ruraux, dans les communautés villageoises et permet des contacts permanents entre les zones rurales et urbaines, et entre le pays et le monde extérieur. Ainsi, le téléphone mobile contribue avec d'autres médias (notamment la télévision par satellite) à une modification de la représentation du monde externe et les rapports au temps et à l'espace en sont affectés. L'exemple des migrants ${ }^{19}$ aujourd'hui est édifiant à ce sujet. Avec un simple mobile et plus encore avec un smartphone, le lien entre eux et ceux qui demeurent encore dans leur communauté d'origine est instantané et permanent. Les images de ceux qui bravent quotidiennement l'exil au péril de leur vie accrochés à leur téléphone sont autant dramatiques que parlantes. Le cellulaire, la messagerie électronique et les réseaux sociaux permettent aujourd'hui à beaucoup de jeunes Africains, et tous «ceux qui n'ont pas les mêmes ressources de mobilité la possibilité de rendre leur vie plus excitante (Cardon, 2013) » tout en respectant les éléments traditionnels de leur société, de mener une vie, qui n'est pas que virtuelle, en prise avec le monde. 
21 C'est un monde devenu tout petit et traversé par toutes sortes de flux, commerciaux, financiers, populations, etc. que les TN rendent paradoxalement proche et lointain à la fois. Ces technologies permettent ainsi aux jeunes africains nés avec le numérique (digital natives $)^{20}$ d'exprimer cet irrépressible désir de monde, de vivre et de voyager comme les autres jeunes des pays développés. Et à défaut de pouvoir circuler librement à travers la planète, ils vivent la mondialisation, ils sont dans la mondialisation, se l'approprient grâce aux TN et contribuent à en redéfinir les contours et le fonctionnement. C'est une position et un rôle nouveaux pour des populations longtemps négligées et marginalisées.

Enfin, contrairement aux décennies précédentes, l'accès et les utilisations des TN sont en grande partie le résultat, non pas d'une stratégie étatique, du volontarisme politique, mais d'appropriations individuelles qui s'appuient et renforcent une dynamique collective de réseau ${ }^{21}$. Et ainsi que le souligne A. Chéneau-Loquay, "on a donc un développement des usages "par le bas » qui est très différent du modèle classique de la délivrance d'infos "par le haut », celui de la radio ou de la TV et qui se rapproche par contre de celui d'Internet qui se développe selon le modèle biologique de l'éponge par capillarité (Chéneau Loquay, op.cité) ». Il y a à l'évidence une véritable spécificité des TN qui, au-delà de la technique elle-même, font jouer aux utilisateurs un rôle très important de partenaires, de chercheurs et de promoteurs de leur développement (Renaud, op.cité). Ils ouvrent de nouvelles opportunités et permettent de nouvelles perspectives pour les économies et des sociétés en développement et notamment africaines. D’autant plus que les potentialités des TN conjuguées aux processus ouverts (logiciels libres, plateformes de connaissances, etc.) pourraient considérablement amplifier l'accès aux ressources en matière de développement humain et contribuer ainsi à développer, renforcer ou élargir le champ des capabilités, au sens que leur donne A. Sen (Sen, 2000). Les TN pourraient alors constituer pour les populations pauvres et démunies, des vecteurs d'acquisition ou de renforcement de leurs capabilités.

Grâce aux TN les individus des PED pourraient alors accéder au pouvoir de décision et de gestion de ressources nécessaires à leur bien-être et à la vie qu'ils souhaitent mener. Ces technologies libèrent en effet les usages et permettent la création d'espaces d'opportunités considérables en jouant un rôle permissif important pour toutes les activités sociales exigeant l'information. Et à ce titre, les TN ouvrent de nouvelles possibilités pour accéder à l'information, les connaissances, la culture, nécessaires au développement humain comme promotion des libertés.

\section{BIBLIOGRAPHIE}

Marc Andreessen, Wall Street Journal, 20/08/2011

Séverine Arsène, « De l'autocensure aux mobilisations ", Revue française de sciences politiques, vol. 6, $n^{\circ}$ 5, 2011, pp. 893-915.

Michael Béchir Ayari, « Non les révolutions tunisiennes et égyptienne ne sont pas des révolutions $2.0 »$, Mouvements, $\mathrm{n}^{\circ}$ 66, 2011, pp. 56-61. 
Jérôme Batout, « Le monde selon Facebook », Le Débat, n 163, 2011, pp. 4-15.

Gérard Berry, Pourquoi et comment le monde devient numérique, Collège de France/ Fayard, Paris, 2009.

Pierre Buhler, « Révolution numérique et ébranlement des États », Commentaire, n 134, 2011, pp. 339-348.

Dominique Cardon, «Liens faibles et liens forts sur les réseaux sociaux », Cahiers Français, $\mathrm{n}^{\circ}$ 372, 2013, pp. 21-34.

Dominique Cardon, La démocratie Internet, Promesses et limites, La république des idées, Seuil, Paris, 2010.

Annie Chéneau-Loquay, «L'Afrique au seuil de la révolution des télécommunications. Les grandes tendances de la diffusion des TIC », Afrique contemporaine, $n^{\circ}$ 234, 2010a, pp. 93-112.

Annie Chéneau-Loquay, Modes d'appropriation innovants en téléphonie mobile en Afrique, MAEE, UIT, Paris, 2010b.

CNUCED, « Rapport 2013 sur l'économie de l'information », https://unctad.org/fr/ PublicationsLibrary/ier2013overview_fr.pdf, (Consulté le 10 novembre 2016).

CNUCED, "Science et technologie pour le développement : Le nouveau paradigme des TIC », 2008, in https://unctad.org/fr/docs/sdteecb20071overview_fr.pdf, (consulté le 12 mars 2016).

Nicoals Curien, Pierre Alain Muet, La société de l'information, Rapport du Conseil d'analyse économique, La Documentation Française, Paris, 2004.

Ahmed Dahmani, « Rente et compromis social en Algérie, vers la rupture ? », Raison présente, $\mathrm{n}$ - 181, 2012, pp. 97-103.

Ahmed Dahmani, « Economie politique de l'Internet au Maghreb : Incertitudes d'une démocratisation du numérique ", In Ahmed Dahmani A. (dir.), La démocratie à l'épreuve de la société numérique, Karthala, Paris, 2007, pp. 297-324.

Elyes Feki, La révolution tunisienne, une révolution numérique ?, Mémoire de Master 2, HEC Paris/Mines Paris Tech, 2011.

Jean-Jacques Gabas (dir.), Société numérique et développement en Afrique, Karthala, Paris, 2004.

Alain Kiyindou A et alii, dir., Quand l'Afrique réinvente la téléphonie mobile, L'Harmattan, Paris, 2015.

Paul Mathias, «Les enfants d'Emile. La mutation du processus de connaissance de l'ère numérique », Futuribles, n 379, 2011,pp. 42-57.

Franck Mermier (dir.), Mondialisation et nouveau médias dans l'espace arabe, Paris, MaisonnneuveLarose, 2003.

Mihoub Mezouaghi (dir.), Le Maghreb dans l'économie numérique, IRMC, Maisonnneuve-Larose, Paris, 2007.

Khadija Mohsen-Finan, Les médias en Méditerranée - Nouveaux médias, monde arabe et relations internationales, Actes Sud, 2009.

Ruth Mireille Manga Edimo, « Les TIC, nouvelles formes d'action politique. Le cas des diasporas camerounaises », Afrique Contemporaine, $\mathrm{n}^{\circ}$ 234, 2010, pp. 127-140.

Julien Nocetti, «Internet, accélérateur des transformations de la Russie », Etudes, $n^{\circ} 418,2013$, pp. 151-160.

OECD, Digital Economy Outlook, 2015. 
OIF : « Rapport sur la Francophonie numérique, 2016 » : https://www.francophonie.org/rapportnumerique-2016.html, (consulté le 10 novembre 2016).

\section{NOTES}

1. Initialement appelées les technologies de l'information et de la communication (TIC), nous utiliserons la nouvelle dénomination donnée par la Banque Mondiale dans son rapport sur le développement dans le monde de 2016. Les TN englobent l'Internet, les téléphones mobiles et tous les autres outils servant à recueillir, stocker, analyser et partager des informations sous forme numérique; in http://documents.worldbank.org/curated/en/527621468195004729/ pdf/102724-WDR-WDR2016Overview-FRENCH-WebResBox-394840B-OUO-9.pdf

2. La première date de la fin du $18 \mathrm{e}$ siècle et se fonde sur les progrès en mécanique et en chimie. La seconde apparait vers la fin du 19e siècle avec la maitrise de l'énergie et l'invention du moteur à explosion. Les deux vont provoquer des séries de transformations socioéconomiques et politiques qui vont profondément bouleverser le monde occidental.

3. Adopté en 2014 par l'UIT, ce programme fixe une série d'objectifs comme l'amélioration de la croissance et surtout le caractère inclusif des TIC.

4. L'indice de développement des TIC est la moyenne de onze indicateurs de base reflétant l'accès aux TIC, leur utilisation et les compétences dans ce domaine, in «Mesurer la société de l'information » : https://www.itu.int/en/ITU-D/Statistics/Documents/publications/misr2017/ MISR2017_ES_F.pdf

5. Gabas Jean Jacques. (dir.), 2004, Société numérique et développement en Afrique, Paris, Karthala.

6. En 2010, selon un rapport de l'ONU sur la réalisation des objectifs du millénaire la croissance des TIC demeure malgré la crise et notamment en Afrique : « en dépit de signes témoignant d'une faiblesse des inves tissements, la récente récession ne semble pas avoir ralenti la croissance au niveau de l'utilisation des services liés aux TIC», Voir ONU, «Objectifs du Millénaire pour le développement, Rapport 2010 [en ligne], http://www.un.org/fr/millenniumgoals/pdf/ report2010.pdf.

7. Les cartes représentent plus de $90 \%$ des ventes des opérateurs de télécommunication.

8. Selon l'UIT, op.cité., dans les pays les moins avancés (PMA), le panier des prix des services mobiles a chuté de moitié, ne représentant plus, fin 2014, que $14 \%$ du PIB/hab. contre $29 \%$ en 2008.

9. On retrouve l'idée au niveau de la Commission économique pour l'Afrique qui affirme: «L'évolution du monde vers l'ère de l'informatique et les innovations technologiques qui ont lieu partout dans le monde depuis ces dernières années, conjuguée à d'autres changements structurels économiques, ont fait rapidement tomber les coûts des technologies de l'information et de la communication. Grâce à cette évolution, combinée aux progrès des télécommunications mondiales et nationales, il est incontestable que l'on peut mettre en place des stratégies adaptées permettant de sauter des étapes pour accélérer le développement du continent », cité par Marchés tropicaux et méditerranéens, juin 2000.

10. La Banque semble avoir révisé son analyse. C'est ainsi que dans son rapport sur le dévelop pement dans le monde de 2016 (les dividendes du numérique), il y est dit que « les techno logies numériques ne constituent en aucun cas un raccourci vers le développement, elles peuvent stimuler ce dernier, voire l'accélérer », op. cit. p. 4.

11. Il faut noter à ce sujet la difficile concré tisation, y compris pour les pays développés, de toutes les potentialités théoriques ouvertes par les TN.

12. Banque mondiale, op. cité.

13. UIT, Mesurer la société de l'information, op.cit. 
14. C.O. Traoré relève qu'il est « fascinant de voir comment des habitants analpha bètes dans les zones rurales s'approprient cette technologie et les changements qu'elle introduit dans leur vie, « les impacts du téléphone mobile sur les communautés en Afrique de l'Ouest, in Alain Kiyindou et alii (eds) Quand l'Afrique réinvente la téléphonie mobile, L'Harmattan, 2015.

15. B.P.S. Akregbou, «Usages du téléphone mobile en public et construction d'une identité chez les jeunes Abidjanais ", in A. Kiyindou, op.cit.

16. Selon le rapport de la Banque mondiale, dans les PED, «près de $70 \%$ des personnes du quintile inférieur de l'échelle économique sont propriétaires d'un mobile », op. cité., p. 3.

17. S. Tietse affirme ainsi dans son étude que « les constructeurs devront tenir compte dans le processus de fabrication du nouveau futur téléphone mobile de l'intégration des « nouveaux arts de faire (De Certeau) et des pratiques décelées à travers des tactiques d'usages prescrits ayant entrainé de nouveaux usages ", "le téléphone portable dans les inte ractions quotidiennes des étudiants d'un site universitaire rural en Afrique: cas de l'Uni versité de Yaoundé II-SOA au Cameroun ", in Alain Kiyindou, op.cité, p. 150.

18. Bernard Steiner traitant du rôle des intermédiaires à l'heure d'Internet au Mali avance que «le courriel autorise une liberté de ton qui, en d'autres circonstances, serait impossible ... l'aspect asynchrone du courriel, qui est celui de l'écrit en général, permet au cadet de s'adresser directement à son aîné malgré l'asymétrie de leur lien. Sur la Toile, les cadets n'ont donc plus besoin d'emprunter la voix d'un tiers pour « régler leurs comptes » avec les aînés, il leur suffit de se cacher derrière l'écran de leur ordinateur ", in "Les intermédiaires, acteurs clés des réseaux Internet transnationaux, Le cas des cybercafés de Bamako », Afrique Contemporaine, 234/2010.

19. Patrick Chamoiseau, Frères migrants, Le Seuil, Paris, 2017.

20. S. Tietse, op. cité.

21. Il est important de noter à cet effet que ce qui distingue la demande de télécommunications de celle des autres biens et services, c'est le fait que les services de communication ne sont pas consommés de façon isolée, mais en réseau. Ce qui implique des interdépendances et des externalités qui affectent la manière de modéliser la consommation de télécoms, mais également une distinction entre accès et usage.

\section{RÉSUMÉS}

Les technologies numériques constituent l'innovation majeure de ces dernières décennies et le principal vecteur de la nouvelle révolution industrielle dans les pays développés. Ces technologies ont radicalement transformé les modes de vie, les façons de produire, les rapports au temps et à l'espace, l'environnement culturel. Quid alors des pays en développement, notamment africains, où ces technologies connaissent une progression fulgurante. Quels sont leurs différents impacts dans ces pays où leur progression dépasse toutes les prévisions. Comment les différents usages et les capacités d'appropriation manifestés par les sociétés africaines nous interpellent pour réfléchir à un nouveau paradigme qui tienne compte de toutes les mutations et changements socioéconomiques et culturels que ces technologies provoquent?

The digital technologies establish the major innovation of these last decades and the main vector of the new industrial revolution in the developed countries. These technologies radically transformed the lifestyles, the manners to produce, reports in the time and in the space, the cultural environment. Quid then developing countries, in particular African, where these 
technologies know a lightning progress. Which are their various impacts in these countries where their progress exceeds all the forecasts. How the various uses and the capacities of appropriation shown by the African companies question us to reflect in new one paradigm which takes into account all the transfers and the socioeconomic and cultural changes which these technologies cause?

Las tecnologías numéricas constituyen la innovación superior de estas últimas décadas y el principal vector de la nueva revolución industrial en los países desarrollados. Estas tecnologías radicalmente transformaron los modos de vida, los modos de producir, los informes(relaciones) al tiempo y al espacio, el entorno(medio ambiente) cultural. Quid entonces países en vías de desarrollo, particularmente africanos, donde estas tecnologías conocen una progresión fulgurante. Cuales son sus diferentes impactos en estos países dónde su progresión sobrepasa todas las previsiones. ¿ Cómo los diferentes usos y las capacidades de apropiación manifestados por las sociedades africanas nos interpelan para reflexionar sobre un nuevo paradigma que tenga en cuenta todas las mudanzas(mutaciones) y los cambios socioeconómicos y culturales que estas tecnologías provocan?

\section{INDEX}

Palabras claves : tecnologías numéricos, mundialización, país en vías de desarrollo, individuo, comunidad, mujer, libertades, capacidad

Mots-clés : technologies numériques, mondialisation, pays en développement, individu, communauté, femme, libertés, capabilités

Keywords : digital technologies, globalization, developing country, individual, community, woman, liberties, capabilities

\section{AUTEUR}

\section{AHMED DAHMANI}

Collège d'études interdisciplinaires (CEI), Université Paris Sud, France 